PROC. OF JSCE

No. 314, Oct. 1981

\title{
AN EDGE CRACK IN A SEMI-INFINITE PLATE WELDED TO A RIGID STIFFENER
}

\author{
By Norio HASEBE*
}

\section{INTRODUCTION}

A part on a boundary of a semi-infinite plate is rigidly stiffened. The semi-infinite plate with a crack originating from an end of the rigid stiffener is analyzed as a mixed boundary value problem. The stiffener is subjected to displacements due to rotation and translation, and a remaining part on the boundary is free. Stress distributions and stress intensity factors are investigated. This is a model of a crack originating from a fixed end of an elastic body subjected to plane forces. This also is a model of a crack originating from an end of rigid stamp with friction subjected to rotation and translation.

Since an infinite stress occurs at the end of the stiffener, a crack may occur. Therefore it seems of interest to investigate the state of stress before and after occurrence of a crack and obtain the stress intensity factor. Stress intensity factors for various different cracks and for various conditions are calculated and methods for obtaining them have also been developed ${ }^{1) \sim 3}$. However no extensive theoretical analyses for a crack originating from an end of stiffener seem to appear.

In this paper the method ${ }^{4}$ by means of a rational mapping function is used for the stress analysis. A mixed boundary value problem is mathematically more difficult than a stress or displacement boundary value problem. Also in this case, the closed solution can be obtained. Therefore the solution is exact for the shape represented by the rational mapping function. The rational mapping function is formed as a sum of fractional expressions. By using a rational mapping function of such type, Okabayashi ${ }^{5)}$ and the author ${ }^{4), 6), 7)}$ have analyzed stress or mixed boundary value problem in a plane elastic problem and a transverse bending problem of

* Member of JSCE, Dr. Eng., Associate professor, Dept. of Civil Engineering, Nagoya, Institute of Technology thin plate. Rational mapping function of a sum of fractional expressions has also been applied for crack problem.

\section{MAPPING FUNCTION}

A rational mapping function that maps a semi-infinite region with an edge crack into a unit circle is formed from an irrational mapping function obtained by Schwarz-Christoffel's transformation. The irrational mapping function is

$$
Z=-i b \sqrt{2} \frac{\sqrt{1+\zeta^{2}}}{1-\zeta}+\frac{a}{2}
$$

The shape on the $z$ plane and the corresponding unit circle are shown in Fig. 1, in which " $a$ " is a length of a stiffener and " $b$ " is a crack length. Imaginary unit " $-i$ " in Eq. (1) is used so that the boundary line of the semi-infinite region is parallel to the $x$ axis. The constant " $a / 2$ " is added so that the $y$ axis passes through the center of the stiffener. Parameter $\alpha(=-i$ in this paper) and $\beta$ are variables to correspond to the point $\mathrm{D}$ and $\mathrm{E}$ respectively. If the parameter $\beta$ is changed, the length of the stiffener $a / b$ can be changed.

A rational mapping function of a sum of fractional expressions from Eq. (1) is formed as the following expression,

$$
Z=\omega(\zeta)=\frac{E_{0}}{1-\zeta}+\sum_{k=1}^{20} \frac{E_{k}}{\zeta_{k}-\zeta}+E_{-1}
$$

Since the procedure for forming the above equation is explained in reference 4), 7), it is not explained here due to the limited space. If
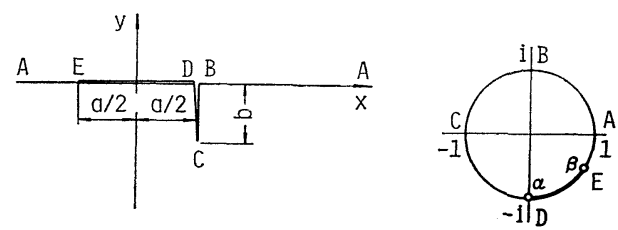

Fig. 1 A Semi-infinite Region with a Crack and a Unit Circle. 
values on the unit circle are substituted into Eq. (2), a diagram can be drawn. Then the difference between Eq. (1) and Eq. (2) can be investigated. Since Eq. (2) is a rational function, the tip of the crack has a round corner. The radius of curvature at the tip of the crack is $\rho / b=0.7846 \times 10^{-12}$ which is very small. Points $\mathrm{B}$ and $\mathrm{D}$ are not sharp corner, but are round corners with radius of curvature $\rho / b=0.0176$. The largest difference between Eq. (1) and Eq. (2) are at points B and D.

\section{METHOD OF ANALYSIS}

The complex variable method $^{8), 9)}$ is used. Since an analytical method using a rational mapping function of a sum of fractional expressions is described in reference 4), only the necessary expressions are shown due to limited space. If the regular complex stress functions in the unit circle are denoted by $\phi(\zeta)$ and $\psi(\zeta)$, then the stress components are

$$
\begin{aligned}
& \sigma_{x}+\sigma_{y}=4 \operatorname{Re}\left[\phi^{\prime}(\zeta) / \omega^{\prime}(\zeta)\right] \\
& \sigma_{y}-\sigma_{x}+2 i \tau_{x y}=2\left\{\omega(\zeta)\left[\phi^{\prime}(\zeta) / \omega^{\prime}(\zeta)\right]^{\prime}\right. \\
& \left.\quad+\psi^{\prime}(\zeta)\right\} / \omega^{\prime}(\zeta) \\
& \sigma_{r}+\sigma_{\theta}=\sigma_{x}+\sigma_{y} \\
& \sigma_{\theta}-\sigma_{r}+2 i \tau_{r \theta}=e^{2 i \beta}\left(\sigma_{y}-\sigma_{x}+2 i \tau_{x y}\right)
\end{aligned}
$$

in which $\operatorname{Re}[$ ] shows the real part of [ ]. Stresses $\sigma_{\theta}, \sigma_{r}$ and $\tau_{r \theta}$ are the components in the curvilinear coordinate expressed by the mapping function $z=\omega(\zeta)$ and $\exp (2 i \beta)=\zeta^{2} \omega^{\prime}(\zeta) /\left[|\zeta|^{2} \omega^{\prime}(\zeta)\right]$.

Resultant forces $X$ and $Y$ in the direction of the respective $x$ and $y$ axes over the arc $\widehat{a b}$ acting on the material to the left of $\widehat{a b}$ are

$$
i(X+i Y)=\left[\phi(\zeta)+\omega(\zeta) \frac{\overline{\phi^{\prime}(\zeta)}}{\overline{\omega^{\prime}(\zeta)}}+\overline{\psi(\zeta)}\right]_{a}^{b}
$$

Resultant moment $R_{m}$ about the origin of forces over the arc $\overparen{a b}$ acting on the material to the left of $\overparen{a b}$ is

$$
\begin{aligned}
R_{m}= & R e\left[\int \psi(\zeta) \omega^{\prime}(\zeta) d \zeta-\omega(\zeta) \psi(\zeta)\right. \\
& \left.-\omega(\zeta) \overline{\omega(\zeta)} \frac{\phi^{\prime}(\zeta)}{\omega^{\prime}(\zeta)}\right]_{a}^{b} . \quad \ldots \ldots \ldots \ldots . . .
\end{aligned}
$$

We shall designate by $M$ the part which the displacements are given, and by $L$ the part which the external forces are given, and by $\Gamma$ the contour $L+M$. In this paper the part of $M$ $\mathrm{DE}$ in Fig. 1 and $L$ is the remaining part EABCD.

The functions $\phi(\zeta)$ and $\psi(\zeta)$ are given by the following expressions,

$$
\phi(\zeta)=-\chi(\zeta) \sum_{k=1}^{20} \frac{\overline{A_{k}} B_{k}}{\chi\left(\zeta_{k}\right)\left(\zeta_{k}-\zeta\right)}+H(\zeta)
$$

in which

$$
\begin{aligned}
& H(\zeta)=M(\zeta)-\frac{\kappa+1}{2 \pi i \kappa} \chi(\zeta) \int_{M} \frac{M(\sigma)}{\chi(\sigma)(\sigma-\zeta)} d \sigma \\
& M(\zeta)=\frac{1}{2 \pi i} \int_{\Gamma} \frac{f(\zeta)}{\sigma-\zeta} d \sigma \\
& f(\sigma)=\left\{\begin{array}{ll}
i \int\left(p_{x}+i p_{y}\right) d s & \text { on } L \\
-2 G(u+i v) & \text { on } M
\end{array}\right\}
\end{aligned}
$$

and

$$
\begin{aligned}
\psi(\zeta)= & \frac{1}{2 \pi i} \int_{\Gamma} \frac{\overline{f(\sigma)}}{\sigma-\zeta} d \sigma-\overline{\phi(0)} \\
& +\frac{\kappa+1}{2 \pi i} \int_{M} \frac{\bar{\phi}(1 / \sigma)}{\sigma-\zeta} d \sigma-\frac{\bar{\omega}(1 / \zeta)}{\omega^{\prime}(\zeta)} \phi^{\prime}(\zeta) \\
& -\sum_{k=1}^{20} \frac{A_{k} \bar{B}_{k} \zeta_{k}^{\prime 2}}{\zeta_{k}^{\prime}-\zeta} \cdot \cdots \cdots \cdots \cdots \cdots \cdots \cdots(10)
\end{aligned}
$$

The function $\chi(\zeta)$ is the Plemelj function $(\zeta-$ $\alpha)^{m}(\zeta-\beta)^{1-m}$ and $m=0.5-i(\ln \kappa) / 2 \pi$. Coefficients $B_{k}=\bar{E}_{k} / \omega^{\prime}\left(\zeta_{k^{\prime}}\right)(k=1,2, \ldots, 20)$ and $\zeta_{k^{\prime}}$ means $\zeta_{k^{\prime}}=1 / \bar{\zeta}_{k}$, the point of reflection of $\zeta_{k}$ with respect to the unit circle. Coefficients $A_{k}, \bar{A}_{k}$ are unknown constants and can be determined by solving 40 numbers of linear equations in this paper. Values $p_{x}(S)$ and $p_{y}(S)$ in Eq. (9) are external forces in the direction of the respective $x$ and $y$ axes. The integral with respect to $S$ shows the integration along the boundary line. Values $u$ are $v$ in Eq. (9) are displacements in the direction of the respective $x$ and $y$ axes. Value $G$ is shear modulus and

$$
\kappa= \begin{cases}3-4 \nu & \text { (plane strain) } \\ (3-\nu) /(1+\nu) & \text { (generalyzed plane stress) }\end{cases}
$$

where $\nu$ is Poisson's ratio.

\section{BOUNDARY CONDITION}

In this paper, the following boundary conditions are considered. Loading condition (A): The stiffener is tilted by $\epsilon$ about the origin (see Fig. 2 (a)).

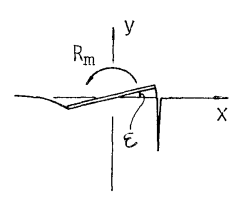

(a) Loading Condition (A).

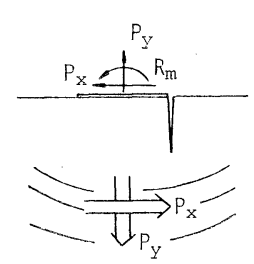

(b) Loading Condition (B).

Fig. 2 


\section{(1) Loading Condition (B)}

The stiffener is translated by the horizontal resultant force $P_{x}$, vertical resultant force $P_{y}$, and the resultant moment $R_{m}$ (necessary in order that the stiffener holds in horizontal position) act (see Fig. 2 (b)).

\section{( 2 ) Loading Condition (A)}

The external force is free on $L$ and the displacement on $M$ is $-2 G(u+i v)=-2 G i \epsilon x \approx$ $-2 G i \epsilon \omega(\zeta)$ since $y \approx 0$ on $M$. The function $M(\zeta)$ in Eq. (8) is

$$
\begin{aligned}
M(\zeta)= & \frac{-G \epsilon E_{0}}{\pi(1-\zeta)} \ln \left(\frac{1-\alpha}{1-\beta} \frac{\zeta-\beta}{\zeta-\alpha}\right) \\
& -\frac{G \epsilon}{\pi} \sum_{k=1}^{20} \frac{E_{k}}{\zeta_{k}-\zeta} \ln \left(\frac{\zeta_{k}-\alpha}{\zeta_{k}-\beta} \frac{\zeta-\beta}{\zeta-\alpha}\right) \\
& -\frac{G \epsilon E_{-1}}{\pi} \ln \frac{\zeta-\beta}{\zeta-\alpha} \quad \ldots \ldots \ldots \ldots \ldots \ldots
\end{aligned}
$$

Equation (11) is substituted into Eq. (7) and the integration involving the Plemelj function must be carried out. The procedure of carrying out the integral is described in reference 10), (p. 67), 11) (p. 18).

The integration of terms involving $\ln [(\zeta-\beta) /$ $(\zeta-\alpha)]$ is tedious, but the integral procedure is briefly explained in reference (4). After all, the complex function $\phi(\zeta)$ is

$$
\begin{aligned}
\phi(\zeta)= & \chi(\zeta)\left[\frac{-2 G i \epsilon E_{0}}{(1+\kappa) \chi(1)(1-\zeta)}\right. \\
& -\frac{2 G i \epsilon}{1+\kappa} \sum_{k=1}^{20} \frac{E_{k}}{\chi\left(\zeta_{k}\right)\left(\zeta_{k}-\zeta\right)} \\
& \left.-\sum_{k=1}^{20} \frac{\bar{A}_{k} B_{k}}{\chi\left(\zeta_{k}\right)\left(\zeta_{k}-\zeta\right)}\right]+\frac{2 G i \epsilon}{1+\kappa} \omega(\zeta)
\end{aligned}
$$

The complex stress function $\psi(\zeta)$ can be determined from Eq. (10), i.e.,

$$
\psi(\zeta)=-\bar{\phi}(1 / \zeta)-\frac{\bar{\omega}(1 / \zeta)}{\omega^{\prime}(\zeta)} \phi^{\prime}(\zeta)
$$

However, since in this case the external force is free on the part of $L$, Eq. (13) can directly be obtained by using the principle of analytic continuation. Since the principle of analytic continuation to determine $\psi(\zeta)$ is described in reference 11) (p. 149), the explanation is omitted. Resultant forces on the stiffener can be calculated from Eq. (4), and in this case is equal to zero. The resultant moment on the stiffener can be calculated from Eq. (5). Upon substituting Eq. (13) into Eq. (5), and using the integration by parts, Eq. (5) can be simplified. Further, upon considering that $\phi(\alpha)=\phi(\beta)=\bar{\phi}(1 / \alpha)=\bar{\phi}(1 /$ $\beta)=0$ and that the value $[\bar{\omega}(1 / \sigma) \omega(\sigma)]_{\alpha}^{\beta}$ is real, the following expression can be obtained,

$$
\left.\begin{array}{rl}
R_{m}= & -\operatorname{Re} \int_{\alpha}^{\beta}\left[\bar{\omega}^{\prime}(1 / \sigma) \chi(\sigma) D(\sigma) / \sigma^{2}\right. \\
& \left.+\omega^{\prime}(\sigma) \bar{\chi}(1 / \sigma) \bar{D}(1 / \sigma)\right] d \sigma \\
= & -\operatorname{Re}\left[\frac{-\kappa}{1+\kappa} \oint \bar{\omega}^{\prime}(\bar{\sigma}) \chi(\sigma) D(\sigma) d \bar{\sigma}\right. \\
& \left.-\frac{1}{1+\kappa} \oint \omega^{\prime}(\sigma) \bar{\chi}(\bar{\sigma}) \bar{D}(\bar{\sigma}) d \sigma\right] \\
= & -\operatorname{Re}\left[\oint \bar{\omega}^{\prime}(1 / \sigma) \chi(\sigma) D(\sigma) \frac{d \sigma}{\sigma^{2}}\right]
\end{array}\right\} \cdots(14)
$$

in which the function $D(\zeta)$ denotes the function inside of [] in Eq. (12). The line integration involving the Plemelj function $\chi(\sigma)$ has been changed to the integration surrounding the branch cut DE. Upon considering that the integrand is the conjugate relation, Eq. (14) can be obtained. The integral can be carried out by using the theorem of residue. Putting $\bar{A}_{k}=$ $E_{k}=0$ in Eq. (12) and Eq. (2), the solution before occurrence of a crack can be obtained. The solution in this case has been obtained without the use of the mapping function ${ }^{10), 11)}$.

\section{( 3 ) Loading Condition (B)}

The problem in this case is the same by translating the semi-infinite plate while the stiffener is kept fixed. Therefore the boundary condition of Eq. (9) is

$$
\begin{aligned}
i \int\left(p_{x}+i p y\right) d s & =0 & & \text { on } E A \\
& =i P_{x}+P_{y} & & \text { on } A B C D \\
u+i v & =0 & & \text { on } D E .
\end{aligned}
$$

The function $M(\zeta)$ in Eq. (8) is

$$
M(\zeta)=\frac{i P_{x}+P y}{2 \pi i} \ln \frac{\zeta-\alpha}{\zeta-1} .
$$

Equation (15) is substituted into Eq. (7) and the integration involving the Plemelj function must be carried out, but it seems that the integral is difficult. However the first derivative $H^{\prime}(\zeta)$ can be obtained (see Appendix I),

$$
\begin{aligned}
H^{\prime}(\zeta)= & \frac{i P_{x}+P_{y}}{2 \pi i(1-\zeta)}(1-\alpha)^{1-m} \\
& \cdot(1-\beta)^{m}(\zeta-\alpha)^{m-1}(\zeta-\beta)^{-m} .
\end{aligned}
$$

Therefore the first derivative of $\phi(\zeta)$ is obtained as

$$
\begin{aligned}
\phi^{\prime}(\zeta)= & -\chi^{\prime}(\zeta) \sum_{k=1}^{20} \frac{\bar{A}_{k} B_{k}}{\chi\left(\zeta_{k}\right)\left(\zeta_{k}-\zeta\right)} \\
& -\chi(\zeta) \sum_{k=1}^{20} \frac{\bar{A}_{k} B_{k}}{\chi\left(\zeta_{k}\right)\left(\zeta_{k}-\zeta\right)^{2}}+H^{\prime}(\zeta)
\end{aligned}
$$

Next, the complex stress function $\psi(\zeta)$ must be determined. 
Since not $\phi(\zeta)$ but $\phi^{\prime}(\zeta)$ is known, $\psi(\zeta)$ can not be determined from Eq. (10). However since it may be considered that the external force is free on the part of $L$ and the displacements are given on $M$, Eq. (13) can also be obtained by using the principle of analytic continuation.

The resultant moment on the stiffener can be calculated from Eq. (5). This resultant moment occurs in order that the stiffener holds in the horizontal position. However since not $\phi(\zeta)$ but $\phi^{\prime}(\zeta)$ is known, Eq. (5) is changed to the expression in which $\phi^{\prime}(\zeta)$ is included by using the integration by parts. By similar procedure to Eq. (14), the following expression can be obtained,

$$
\left.\begin{array}{c}
R_{m}=-\operatorname{Re} \int_{\alpha}^{\beta}\left[\omega(\sigma) \bar{\phi}^{\prime}(1 / \sigma) / \sigma^{2}\right. \\
\left.+\bar{\omega}(1 / \sigma) \phi^{\prime}(\sigma)\right] d \sigma \\
=-\operatorname{Re}\left[\oint \bar{\omega}(1 / \sigma) \phi^{\prime}(\sigma) d \sigma\right]
\end{array}\right\}
$$

When $\alpha=\beta$ in Eq. (17), the solution for concentrated forces $P_{x}$ and $P_{y}$, acting without the stiffener, can be obtained.

\section{EXAMPLE OF ANALYSIS}

Examples of stress distributions for loading condition (A) are shown in Fig. 3. When value $\alpha$ is fixed at " $-i$ ", value $\beta$ varies with value $a / b$.
Fig. 3(a) shows stress distributions before occurrence of a crack while Fig. 3(b) shows the stress distributions on the boundary line and on the $x / a=1 / 2$ after occurrence of a crack with length $b / a=1$. Fig. 3(c) and 3(d) show stresses $\sigma_{y}\left(\approx \sigma_{r}\right)$ and $\tau_{x y}\left(\approx-\tau_{r \theta}\right)$ on the stiffener. The dashed lines in Fig. 3(c) and 3(d) denote stresses $\sigma_{y}\left(=\sigma_{r}\right)$ and $\tau_{x y}\left(=-\tau_{r \theta}\right)$ before occurrence of a crack. The following relation holds between the stress components $\sigma_{\theta}$ and $\sigma_{r}$ on the stiffener,

$$
\sigma_{\theta} / \sigma_{r}=(3-\kappa) /(1+\kappa) .
$$

Upon substituted Eq. (13) into Eq. (3) and considering $\bar{\sigma}=1 / \sigma$ and $\chi(1 / \sigma)^{-}=-\kappa \chi(1 / \sigma)^{+}$on the stiffener, Eq. (19) can be derived. Though the first derivatives of stress function have the factor $(\zeta-\alpha)^{m-1}(\zeta-\beta)^{-m}$ of which $m$ is a complex number, the stress distribution at the corner near the stiffener in Fig. 3(b) does not show the nature of the oscillation. The stress singularities in the angular corner for the various boundary conditions are investigated by Williams M. L. ${ }^{12)}$. The stress behaviour at the corner near the stiffener in this paper corresponds to the behaviour in the corner with 90 degree for the boundary condition, clamped-free. The first root of the characteristic equation for the stress singularity is real root. Therefore the stress distribution at the corner does not show the nature of the oscillation. This fact coincides with the preceding fact. Fig. 4 shows an example of stress distributions for the case $P_{x} \neq 0$ and

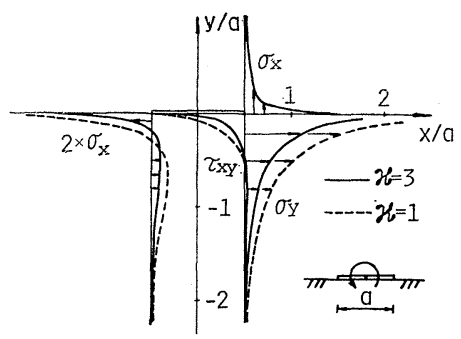

(a)

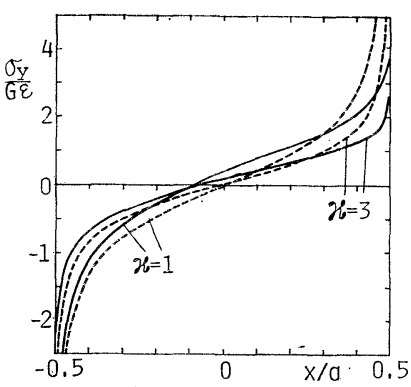

(c)

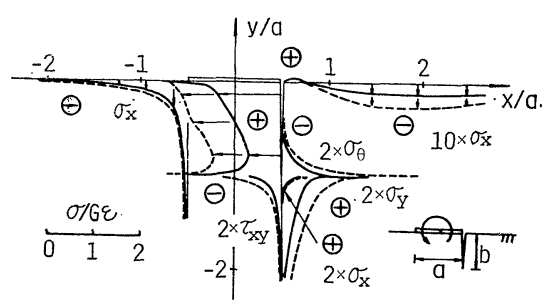

(b)

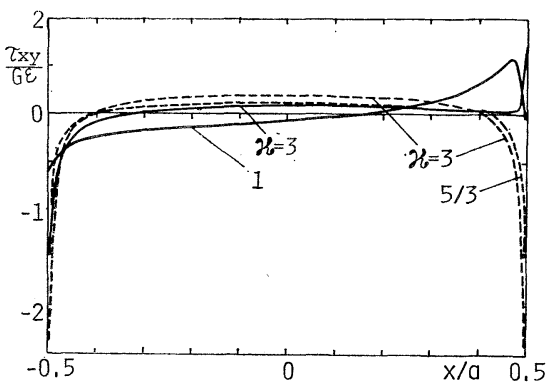

(d)

Fig. 3 Stress Distributions for Loading Condition (A). 


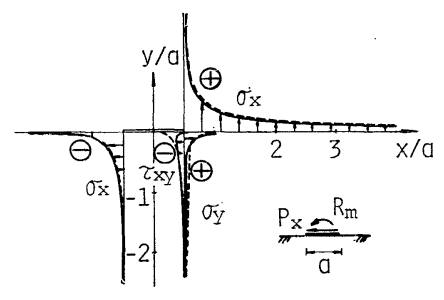

(a)

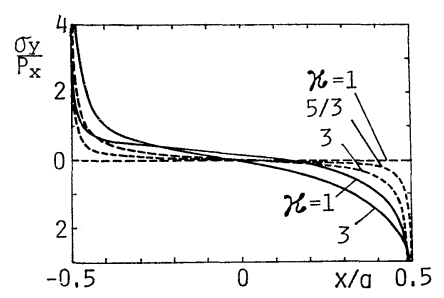

(c)

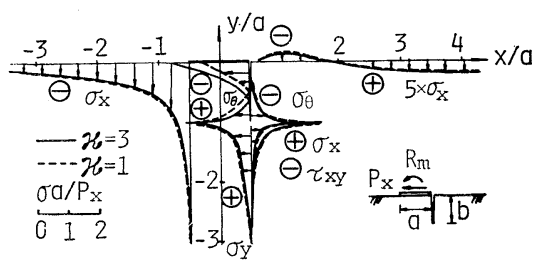

(b)

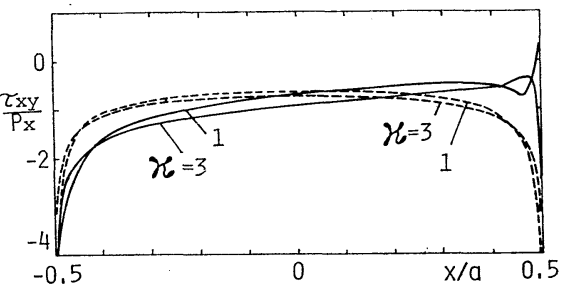

(d)

Fig. 4 Stress Distributions in the Case $P_{x} \neq 0$ and $P_{y}=0$ for Loading Condition (B).

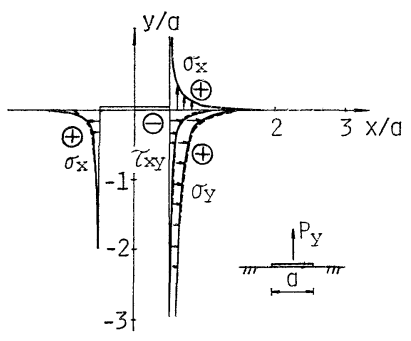

(a)

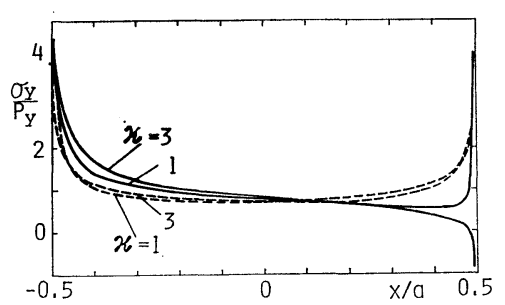

(c)

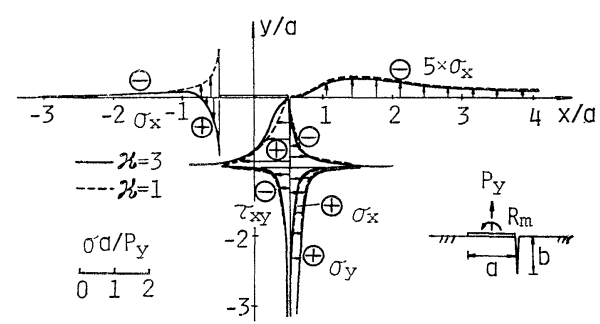

(b)

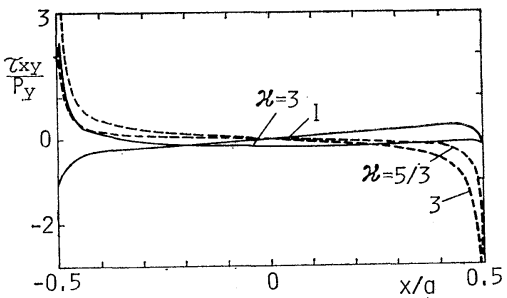

(d)

Fig. 5 Stress Distributions in the Case $P_{x}=0$ and $P_{y} \neq 0$ for Loading Condition (B).

$P_{y}=0$ in loading condition (B), and Fig. 5 is that of the case $P_{x}=0$ and $P_{y} \neq 0$. Fig. 4(a) and $\mathbf{5}(\mathrm{a})$ show stress distributions before occurrence of a crack. Fig. $\mathbf{4}(\mathrm{b})$ and $\mathbf{5}$ (b) show stress distributions after occurrence of a crack with length $b / a=1$. Fig. 4(c), (d) and Fig. 5(c), (d) show stresses $\sigma_{y}\left(\approx \sigma_{r}\right)$ and $\tau_{x y}\left(\approx-\tau_{r \theta}\right)$ on the stiffener. The dashed lines in the figures denote stresses $\sigma_{y}\left(=\sigma_{r}\right)$ and $\tau_{x y}\left(=-\tau_{r \theta}\right)$ before occurrence of a crack. The resultant moment $R_{m} /$ $\left(a^{2} G \epsilon\right)$ on the stiffener for loading condition (A) is shown in Fig. 6(a) and Table 1 for some values of $\kappa$. The counterclockwise sense about the origin is shown as positive moment. The values of resultant moment are shown versus $b / a$ when $0 \leq b / a \leq 1$, and versus $a / b$ when $0 \leq a / b \leq 1$. This resultant moment is required in order to tilt the stiffener. The value of $R_{m} /\left(a^{2} G \epsilon\right)$ for $b / a=0$ is the one before occurrence of a crack. The value can be calculated from $R_{m} /\left(a^{2} G \epsilon\right)=$ $\pi\left\{1+4[(\ln \kappa) /(2 \pi)]^{2}\right\} /[2(1+\kappa)]$. When the length of the stiffener $a / b$ is shorten, values of the resultant moment may be influenced by the roundness of the corner at the point $D$ represented by the rational mapping function. Therefore values of the resultant moment may become 


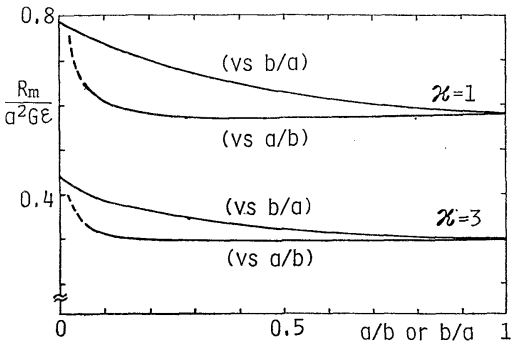

(a)

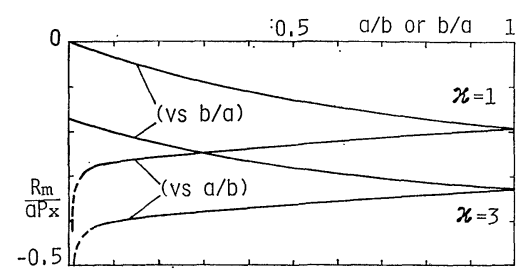

(b)

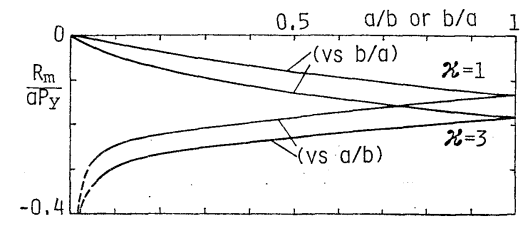

(c)

Fig. 6 Resultant Moments for the Respective Loading Conditions.

Table 1 Resultant Moments $R_{m} /\left(a^{2} G \varepsilon\right)$.

\begin{tabular}{c|c|c|c|c|c|c}
\hline \multicolumn{2}{c|}{$\kappa$} & 3.0 & 2.5 & 2.0 & $5 / 3$ & 1.0 \\
\hline \multirow{5}{*}{$b / a$} & 0.0 & 0.441 & 0.487 & 0.549 & 0.605 & 0.785 \\
& 0.1 & 0.384 & 0.429 & 0.492 & 0.548 & 0.733 \\
& 0.2 & 0.357 & 0.401 & 0.461 & 0.515 & 0.693 \\
& 0.4 & 0.328 & 0.368 & 0.424 & 0.474 & 0.638 \\
& 0.6 & 0.312 & 0.351 & 0.404 & 0.451 & 0.607 \\
& 0.8 & 0.303 & 0.341 & 0.392 & 0.439 & 0.589 \\
& 1.0 & 0.298 & 0.335 & 0.386 & 0.431 & 0.579 \\
\hline \multirow{5}{*}{$a / b$} & 0.8 & 0.295 & 0.332 & 0.381 & 0.426 & 0.572 \\
& 0.6 & 0.293 & 0.329 & 0.378 & 0.423 & 0.567 \\
& 0.4 & 0.292 & 0.329 & 0.378 & 0.422 & 0.567 \\
& 0.2 & 0.298 & 0.335 & 0.385 & 0.430 & 0.578 \\
& 0.1 & 0.309 & 0.348 & 0.400 & 0.447 & 0.602 \\
& 0.05 & 0.332 & 0.373 & 0.429 & 0.479 & 0.648 \\
\hline
\end{tabular}

different from those in the case of the sharp corner at the point $\mathrm{D}$ when $a / b$ is very small. The resultant moment $R_{m} /\left(a P_{x}\right)$ about the origin on the stiffener for the case $P_{x} \neq 0$ and $P_{y}=0$ in loading condition (B) is shown in Fig. 6(b) and Table 2. This resultant moment is required in order that the stiffener holds in the horizontal position. The value of $R_{m} /\left(a P_{x}\right)$ for $b / a=0$ is the one before occurrence of a crack. The value can be calculated from $R_{m} /\left(a P_{x}\right)=$
Table 2 Resultant Moments $R_{m} /\left(a P_{x}\right)$.

\begin{tabular}{c|c|c|c|c|c|c}
\hline \multicolumn{2}{c}{$\kappa$} & 3.0 & 2.5 & 2.0 & $5 / 3$ & 1.0 \\
\hline \multirow{6}{*}{$b / a$} & 0.0 & -0.175 & -0.146 & -0.110 & -0.081 & 0.0 \\
& 0.1 & -0.204 & -0.177 & -0.144 & -0.116 & -0.034 \\
& 0.2 & -0.228 & -0.202 & -0.170 & -0.143 & -0.065 \\
& 0.4 & -0.265 & -0.241 & -0.210 & -0.185 & -0.113 \\
& 0.6 & -0.292 & -0.269 & -0.240 & -0.216 & -0.148 \\
& 0.8 & -0.312 & -0.289 & -0.261 & -0.237 & -0.172 \\
& 1.0 & -0.326 & -0.304 & -0.276 & -0.253 & -0.190 \\
\hline \multirow{6}{*}{$a / b$} & 0.8 & -0.340 & -0.317 & -0.289 & -0.267 & -0.205 \\
& 0.6 & -0.354 & -0.331 & -0.304 & -0.282 & -0.220 \\
& 0.4 & -0.369 & -0.346 & -0.319 & -0.295 & -0.236 \\
& 0.2 & -0.386 & -0.363 & -0.335 & -0.312 & -0.252 \\
& 0.1 & -0.400 & -0.376 & -0.348 & -0.325 & -0.265 \\
& 0.05 & -0.416 & -0.391 & -0.362 & -0.339 & -0.279 \\
\hline 1
\end{tabular}

Table 3 Resultant Moments $R_{m} /\left(a P_{y}\right)$.

\begin{tabular}{c|c|c|c|c|c|c}
\hline \multicolumn{1}{c}{$\kappa$} & 3.0 & 2.5 & 2.0 & $5 / 3$ & 1.0 \\
\hline \multirow{6}{*}{$b / a$} & 0.1 & -0.047 & -0.042 & -0.036 & -0.032 & -0.019 \\
& 0.2 & -0.075 & -0.068 & -0.060 & -0.054 & -0.037 \\
& 0.4 & -0.115 & -0.107 & -0.097 & -0.089 & -0.070 \\
& 0.6 & -0.144 & -0.135 & -0.125 & -0.117 & -0.097 \\
& 0.8 & -0.165 & -0.156 & -0.146 & -0.138 & -0.118 \\
& 1.0 & -0.182 & -0.173 & -0.163 & -0.155 & -0.135 \\
\hline \multirow{6}{*}{$a / b$} & 0.8 & -0.198 & -0.189 & -0.178 & -0.170 & -0.151 \\
& 0.6 & -0.216 & -0.207 & -0.196 & -0.188 & -0.170 \\
& 0.4 & -0.238 & -0.228 & -0.218 & -0.210 & -0.191 \\
& 0.2 & -0.267 & -0.257 & -0.246 & -0.238 & -0.219 \\
& 0.1 & -0.296 & -0.286 & -0.275 & -0.266 & -0.247 \\
& 0.05 & -0.340 & -0.330 & -0.318 & -0.310 & -0.289 \\
\hline
\end{tabular}

$-\ln \kappa /(2 \pi)$. In Fig. 6(c) and Table 3, the resultant moment $R_{m} /\left(a P_{y}\right)$ about the origin on the stiffener for the case $P_{y} \neq 0$ and $P_{x}=0$ in loading condition (B) is shown. This resultant moment is also required in order that the stiffener holds in the horizontal position.

\section{STRESS INTENSITY FACTOR}

Stress intensity factors $K_{\mathrm{I}}$ and $K_{\mathrm{II}}$ of the opening mode and sliding mode can be calculated from the expression,

$$
\begin{aligned}
K_{\mathrm{I}}-i K_{\mathrm{II}}= & 2 \sqrt{2 \pi} \lim _{\zeta \rightarrow \zeta_{1}}\{[\omega(\zeta) \\
& \left.\left.-\omega\left(\zeta_{1}\right)\right] e^{-i \delta}\right\}^{0.5} \phi^{\prime}(\zeta) / \omega^{\prime}(\zeta) \\
= & 2 \sqrt{\pi} e^{-0.5 i \delta} \phi^{\prime}\left(\zeta_{1}\right) / \sqrt{\omega^{\prime \prime}\left(\zeta_{1}\right)}
\end{aligned}
$$

in which $\zeta_{1}$ is a value of $\zeta$ on the unit circle corresponding to the tip of a crack. The value $\delta$ is an angle between the direction of a crack and the $x$ axis. In this paper, $\zeta_{1}=-1$ and $\delta=$ $-\pi / 2$.

Nondimensional stress intensity factors are used for loading condition (A) and loading condition (B). For loading condition (A), nondimensional stress intensity factors are 
$F_{1 a}+i F_{2 a}=\left(K_{\mathrm{I}}+i K_{\mathrm{II}}\right) /(G \epsilon \sqrt{\pi a}): 0 \leq b / a \leq 1$

$F_{1 b}+i F_{2 b}=\left(K_{\mathrm{I}}+i K_{\text {II }}\right) /(G \epsilon \sqrt{\pi b}): 0 \leq a / b \leq 1$.

For loading condition (B), nondimensional



(a)

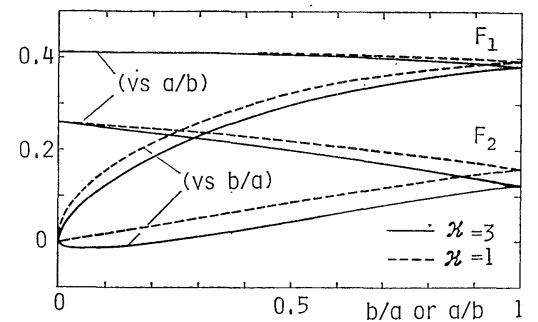

(b)

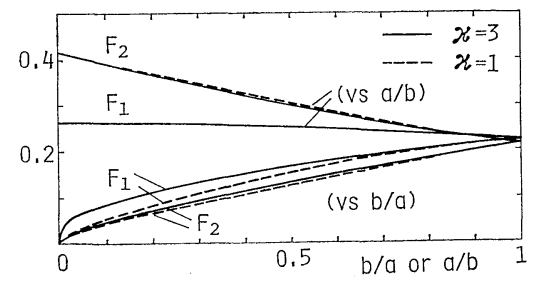

(c)

Fig. 7 Nondimensional Stress Intensity Factors for the Respective Loading Conditions. stress intensity factors for the case $P_{x} \neq 0$ and $P_{y}=0$, and the case $P_{x}=0$ and $P_{y} \neq 0$ are

$$
F_{1}+i F_{2}=\left(K_{\mathrm{I}}+i K_{\mathrm{II}}\right) \sqrt{b} /\left\{\begin{array}{l}
P_{X} \\
P_{Y}
\end{array}\right\} \sqrt{\pi}:
$$

$0 \leq b / a \leq 1$ and $0 \leq a / b \leq 1$.

In Fig. 7(a) and Table 4, the value of the nondimensional stress intensity factor for loading condition (A) are shown. In Fig. 7(b) and Table $\mathbf{5}$, nondimensional stress intensity factors for the case $P_{x} \neq 0$ and $P_{y}=0$ in loading condition (B) are shown. In Fig. 7(c) and Table 6, nondimensional stress intensity factors for the case $P_{x}=0$ and $P_{y} \neq 0$ in loading condition (B) are shown. It is noticed from Eq. (7.a), (7.b) and (7.c) that stress intensity factors in loading condition (A) depend on values of $\kappa$ more than the case of loading condition (B). In Fig. 7(b), 7(c) and Table 5, 6, the values of $F_{1}$ and $F_{2}$ for $a / b=0$ are the nondimensional stress intensity factors for the respective concentrated load $P_{x}$ and $P_{y}$ acting at the point $\mathrm{D}$.

\section{CONCLUSION}

Crack dealed in this paper may not always occur at the right angle to the boundary line. However, the very short crack seems to occur at the angle near the right angle because the maximum stress occurs along the free boundary line at the neighbourhood of the end of the stiffener (See Fig. 3(a), 4(a), 5(a). One elastic investigation regarding to the direction of occurrence of crack can be carried out by means of the mapping function used for the analysis of the oblique crack on the boundary [13].

Since the first derivative Eq. (16) of $H(\zeta)$ for

Table 4 Nondimensional Stress Intensity Factors for Loading Condition (A).

\begin{tabular}{|c|c|c|c|c|c|c|c|c|c|c|}
\hline$\kappa$ & 3.0 & 2.5 & 2.0 & $5 / 3$ & 1.0 & 3.0 & 2.5 & 2.0 & $5 / 3$ & 1.0 \\
\hline$b / a$ & \multicolumn{5}{|c|}{$F_{1 a}$} & \multicolumn{5}{|c|}{$F_{2 a}$} \\
\hline 0.001 & 0.6812 & 0.7087 & 0.7149 & 0.6919 & 0.4542 & 0.1778 & 0.2309 & 0.2942 & 0.3405 & 0.4131 \\
\hline 0.01 & 0.4819 & 0.4989 & 0.5102 & 0.5099 & 0.4470 & 0.2360 & 0.2714 & 0.3138 & 0.3460 & 0.4113 \\
\hline 0.05 & 0.3112 & 0.3310 & 0.3546 & 0.3730 & 0.4160 & 0.2408 & 0.2682 & 0.3025 & 0.3305 & 0.4030 \\
\hline 0.1 & 0.2376 & 0.2581 & 0.2849 & 0.3081 & 0.3789 & 0.2275 & 0.2528 & 0.2853 & 0.3129 & 0.3916 \\
\hline 0.2 & 0.1660 & 0.1847 & 0.2103 & 0.2335 & 0.3109 & 0.2011 & 0.2243 & 0.2551 & 0.2822 & 0.3657 \\
\hline 0.4 & 0.0968 & 0.1099 & 0.1280 & 0.1447 & 0.2021 & 0.1584 & 0.1781 & 0.2049 & 0.2290 & 0.3073 \\
\hline 0.6 & 0.0598 & 0.0682 & 0.0799 & 0.0907 & 0.1277 & 0.1253 & 0.1415 & 0.1637 & 0.1838 & 0.2498 \\
\hline 0.8 & 0.0376 & 0.0429 & 0.0503 & 0.0571 & 0.0802 & 0.0996 & 0.1127 & 0.1305 & 0.1468 & 0.2003 \\
\hline 1.0 & 0.0241 & 0.0274 & 0.0321 & 0.0363 & 0.0507 & 0.0798 & 0.0903 & 0.1047 & 0.1177 & 0.1606 \\
\hline$a / b$ & \multicolumn{5}{|c|}{$F_{1 b}$} & \multicolumn{5}{|c|}{$F_{2 b}$} \\
\hline 0.8 & 0.0127 & 0.0144 & 0.0168 & 0.0189 & 0.0262 & 0.0551 & 0.0623 & 0.0721 & 0.0811 & 0.1105 \\
\hline 0.6 & 0.0049 & 0.0055 & 0.0064 & 0.0071 & 0.0097 & 0.0325 & 0.0367 & 0.0425 & 0.0476 & 0.0647 \\
\hline 0.4 & 0.0010 & 0.0011 & 0.0012 & 0.0014 & 0.0018 & 0.0145 & 0.0164 & 0.0189 & 0.0212 & 0.0286 \\
\hline 0.2 & 0.00001 & 0.00001 & 0.00001 & 0.00001 & -0.00000 & 0.0035 & 0.0040 & 0.0046 & 0.0051 & 0.0068 \\
\hline 0.1 & -0.00001 & -0.00001 & -0.00001 & -0.00001 & -0.00001 & 0.0009 & 0.0010 & 0.0011 & 0.0013 & 0.0017 \\
\hline
\end{tabular}


Table 5 Nondimensional Stress Intensity Factors in the Case $P_{x} \neq 0$ and $P_{y}=0$ for Loading Condition (B).

\begin{tabular}{|c|c|c|c|c|c|c|c|c|c|c|c|}
\hline & & \multicolumn{5}{|c|}{$F_{1}$} & \multicolumn{5}{|c|}{$F_{2}$} \\
\hline & $\kappa$ & 3.0 & 2.5 & 2.0 & $5 / 3$ & 1.0 & 3.0 & 2.5 & 2.0 & $5 / 3$ & 1.0 \\
\hline \multirow{8}{*}{$b / a$} & 0.001 & 0.0027 & 0.0054 & 0.0085 & 0.0107 & 0.0151 & -0.0047 & -0.0040 & -0.0029 & -0.0018 & 0.0012 \\
\hline & 0.01 & 0.0260 & 0.0307 & 0.0359 & 0.0398 & 0.0479 & -0.0110 & -0.0087 & -0.0056 & -0.0030 & 0.0039 \\
\hline & 0.1 & 0.1241 & 0.1296 & 0.1359 & 0.1408 & 0.1531 & -0.0127 & -0.0074 & -0.0009 & 0.0044 & 0.0180 \\
\hline & 0.2 & 0.1880 & 0.1931 & 0.1992 & 0.2041 & 0.2170 & -0.0013 & 0.0049 & 0.0124 & 0.0185 & 0.0344 \\
\hline & 0.4 & 0.2740 & 0.2783 & 0.2835 & 0.2877 & 0.2997 & 0.0320 & 0.0387 & 0.0469 & 0.0535 & 0.0712 \\
\hline & 0.6 & 0.3281 & 0.3315 & 0.3357 & 0.3392 & 0.3490 & 0.0683 & 0.0749 & 0.0830 & 0.0896 & 0.1074 \\
\hline & 0.8 & 0.3617 & 0.3643 & 0.3675 & 0.3702 & 0.3778 & 0.1012 & 0.1075 & 0.1151 & 0.1213 & 0.1383 \\
\hline & 1.0 & 0.3822 & 0.3841 & 0.3865 & 0.3885 & 0.3942 & 0.1286 & 0.1343 & 0.1413 & 0.1470 & 0.1627 \\
\hline \multirow{5}{*}{$a / b$} & 0.8 & 0.3967 & 0.3980 & 0.3997 & 0.4011 & 0.4050 & 0.1549 & 0.1600 & 0.1662 & 0.1712 & 0.1851 \\
\hline & 0.6 & 0.4075 & 0.4083 & 0.4092 & 0.4099 & 0.4120 & 0.1843 & 0.1884 & 0.1934 & 0.1975 & 0.2086 \\
\hline & 0.4 & 0.4129 & 0.4132 & 0.4135 & 0.4137 & 0.4144 & 0.2140 & 0.2168 & 0.2202 & 0.2230 & 0.2306 \\
\hline & 0.2 & 0.4134 & 0.4134 & 0.4134 & 0.4134 & 0.4134 & 0.2402 & 0.2416 & 0.2432 & 0.2446 & 0.2482 \\
\hline & 0.0 & 0.4128 & 0.4128 & 0.4128 & 0.4128 & 0.4128 & 0.2613 & 0.2613 & 0.2613 & 0.2613 & 0.2613 \\
\hline
\end{tabular}

Table 6 Nondimensional Stress Intensity Factors in the Case $P_{x}=0$ and $P_{y} \neq 0$ for Loading Condition (B).

\begin{tabular}{|c|c|c|c|c|c|c|c|c|c|c|c|}
\hline & & \multicolumn{5}{|c|}{$F_{1}$} & \multicolumn{5}{|c|}{$F_{2}$} \\
\hline & $\kappa$ & 3.0 & 2.5 & 2.0 & $5 / 3$ & 1.0 & 3.0 & 2.5 & 2.0 & $5 / 3$ & 1.0 \\
\hline \multirow{8}{*}{$b / a$} & 0.002 & 0.0201 & 0.0190 & 0.0169 & 0.0147 & 0.0065 & 0.0036 & 0.0047 & 0.0057 & 0.0062 & 0.0059 \\
\hline & 0.02 & 0.0513 & 0.0473 & 0.0419 & 0.0369 & 0.0211 & 0.0183 & 0.0196 & 0.0206 & 0.0208 & 0.0187 \\
\hline & 0.1 & 0.0894 & 0.0839 & 0.0767 & 0.0707 & 0.0525 & 0.0492 & 0.0496 & 0.0493 & 0.0485 & 0.0435 \\
\hline & 0.2 & 0.1144 & 0.1094 & 0.1031 & 0.0979 & 0.0825 & 0.0744 & 0.0740 & 0.0729 & 0.0715 & 0.0652 \\
\hline & 0.4 & 0.1526 & 0.1495 & 0.1458 & 0.1427 & 0.1339 & 0.1146 & 0.1139 & 0.1126 & $0.111 \mathrm{I}$ & 0.1051 \\
\hline & 0.6 & 0.1833 & 0.1817 & 0.1799 & 0.1783 & 0.1742 & 0.1507 & 0.1502 & 0.1494 & 0.1484 & 0.1443 \\
\hline & 0.8 & 0.2068 & 0.2061 & 0.2054 & 0.2048 & 0.2032 & 0.1835 & 0.1835 & 0.1833 & 0.1829 & 0.1807 \\
\hline & 1.0 & 0.2237 & 0.2235 & 0.2234 & 0.2233 & 0.2231 & 0.2123 & 0.2127 & 0.2130 & 0.2131 & 0.2124 \\
\hline \multirow{6}{*}{$a / b$} & 0.8 & 0.2378 & 0.2379 & 0.2381 & 0.2382 & 0.2388 & 0.2423 & 0.2431 & 0.2438 & 0.2442 & 0.2447 \\
\hline & 0.6 & 0.2506 & 0.2508 & 0.2511 & 0.2514 & 0.2522 & 0.2794 & 0.2804 & 0.2814 & 0.2821 & 0.2836 \\
\hline & 0.4 & 0.2596 & 0.2598 & 0.2600 & 0.2601 & 0.2606 & 0.3226 & 0.3235 & 0.3246 & 0.3254 & 0.3271 \\
\hline & 0.2 & 0.2629 & 0.2629 & 0.2630 & 0.2630 & 0.2631 & 0.3680 & 0.3686 & 0.3692 & 0.3697 & 0.3708 \\
\hline & 0.1 & 0.2629 & 0.2629 & 0.2629 & 0.2629 & 0.2629 & 0.3900 & 0.3903 & 0.3907 & 0.3909 & 0.3915 \\
\hline & 0.001 & 0.2628 & 0.2628 & 0.2628 & 0.2628 & 0.2628 & 0.4111 & 0.4111 & 0.4111 & 0.4112 & 0.4112 \\
\hline
\end{tabular}

the concentrated load was known, analysis for the fundamental loading has become possible. Equation (19) holds between the stress components $\sigma_{\theta}$ and $\sigma_{r}$ on the rigid stiffener. Stress intensity factors can be obtained with accuracy for both the small values of $b / a$ and the small values of $a / b$. Stress intensity factors of crack occurring at the right angle to the boundary line can be calculated for the arbitrary loads by the combination of the loading condition (A) and (B). Values of resultant moment in the loading condition (A) is larger in the case of $\kappa=1$ than $\kappa=3$, i.e. Poisson's ratio increases values of resultant moment. Stress intensity factors in the loading condition (A) are larger in the case of $\kappa=1$ than $\kappa=3$ except values of $F_{1}$ of the vary short crack. Values of resultant moment in the loading condition (B) is larger in the case of $\kappa=3$ than $\kappa=1$. Values of $F_{1}$ in the case of $P_{X} \neq 0$ and $P_{Y}=0$ is larger in the case of $\kappa=1$ than $\kappa=3$, but the property is reversed in the case of $P_{X}=0$ and $P_{Y} \neq 0$.
Stress intensity factors for the concentrated forces $P_{X}$ and $P_{Y}$ acting at the corner $\mathrm{D}$ have been obtained as the special case.

\section{APPENDIX DERIVATION OF EQ. (16)}

The first derivative of the complex stress function $\Omega(z)$ for the semi-infinite plate (i.e., before occurrence of a crack) in the case of the loading condition (B) is obtained without the use of the mapping function in reference 10) (p. 93), 11) (p. 63),

$$
\Omega^{\prime}(z)=\frac{i p_{x}+p_{y}}{2 \pi i}\left(z-z_{1}\right)^{m-1}\left(z-z_{2}\right)^{-m} .
$$

The values $z$ and $z_{2}$ are the coordinates at the ends of the stiffener (see Fig. 8). The following mapping function that maps the semiinfinite region into the unit circle is used,

$$
z=\omega(\zeta)=E_{0} /(1-\zeta)+E_{-1}
$$

If Eq. (20) is represented by the variable $\zeta$, upon 




Fig. 8 A Semi-Infinite Region and the Unit Circle.

substituting Eq. (21) into Eq. (20), the following expression can be obtained,

$$
\begin{aligned}
\Omega^{\prime}(z)= & \frac{i p_{x}+p_{y}}{2 \pi i E_{0}}(1-\alpha)^{1-m}(1-\beta)^{m} \\
& \cdot(1-\zeta)(\zeta-\alpha)^{m-1}(\zeta-\beta)^{-m}
\end{aligned}
$$

in which $\alpha$ and $\beta$ are the coordinates on the unit circle corresponding to $z_{1}$ and $z_{2}$.

Since $\Omega^{\prime}(z)=\phi^{\prime}(\zeta) / \omega^{\prime}(\zeta)$, the following expression can be obtained from Eq. (21) and Eq. (22),

$$
\begin{aligned}
& \phi^{\prime}(\zeta)=\frac{i P_{x}+P_{y}}{2 \pi i(1-\zeta)}(1-\alpha)^{1-m}(1-\beta)^{m} \\
& \cdot(\zeta-\alpha)^{m-1}(\zeta-\beta)^{-m} \text {. }
\end{aligned}
$$

The first derivative of the function $H(\zeta)$ that is obtained by substituting Eq. (15) into Eq. (7) must equal to Eq. (23). Therefore Eq. (16) can be obtained.

\section{REFERENCES}

1) Sih, G. C.: Handbook of Stress Intensity Factors, Bethlehem, 1973.

2) Sih, G. C. (ed): Mechanics of Fracture, Vol. 1 Noordhoff International Publishing, Ltd., Leyden, 1973.

3) Tada, H.: The Stress Analysis of Crack Handbook, Del Research Corporation, Pennsylvania, 1973.

4) Hesebe, N.: "Uniform tension of a semi-infinite plate with a crack at an end of a stiffened edge," Ingenieur-Archiv 48, pp. 129 141, 1979.

5) Okabayashi, M.: The solution of two dimensional elastic problem where the region is conformally mapped on the semi-infinite plane by the rational function and its application to the infinite thin plate with wedge-shape slit, proceedings of the Japan Society of Civil Engineers, 119, pp. 10 18, 1965.

6) Hasebe, N. and Y. Horiuchi: Stress analysis for a strip with semi-elliptical notches or cracks on both sides by means of rational mapping function, Ingenieur-Archiv 47, pp. 169 179, 1978.

7) Hasebe, N.: Bending of strip with semielliptic notches or cracks, Journal of Engineering Mechanical Division, ASCE, Vol. 104, No. EM6, Proc. Paper 14248, pp. 1433 1450, Dec., 1978.

8) Muskhelishvili, N. I.: Some Basic Problems of the Mathematical Theory of Ealsticity, 2nd English ed., Noordhoff International Publishing, Ltd., the Netherlands, 1963.

9) Mikhlin, S. G.: Integral Enquations and Their Applications to Certain Problems in Mechanics, Mathematical Physics and Technology, 2nd. ed., Pergamon Press, Oxford, 1964.

10) Milne-Thomson, L. M.: Plane Elastic Systems, Springer-Verlag, Berlin, 1968.

11) England, A. H.: Complex Variable Methods in Elasticity, 1st. ed., Wiley-Interscience, London, 1971.

12) William, M. L.: Stress singularities resulting from various boundary conditions is angular corners of plates extension, Journal of Applied Mechanics, pp. 526 528, 1952.

13) Hasebe, N., and S. Inohara: Stress Analysis of a Semi-Infinite Plate with an Oblique Edge Crack, Ingenieur-Archiv 49, pp. 51 62, 1980.

(Received June 13, 1980) 